\title{
PowerDoW (Power Digital Offset Weightage): Video Content- Adaptation (VCA) Profiling in Smartphone Devices for Energy Efficiency
}

\author{
Muhammad Hanif Jofri ${ }^{\mathrm{a}, 1}$, Muharman Lubis ${ }^{\mathrm{b}}$, Mohd Farhan Md Fudzee ${ }^{\mathrm{a}, 2}$, Shahreen Kasim ${ }^{\mathrm{a}, 3}$, \\ Mohd Norasri Ismail ${ }^{\mathrm{a}, 4}$, Deden Witarsyah ${ }^{\mathrm{c}}$ \\ ${ }^{a}$ Faculty of Computer Science and Information Technology, Universiti Tun Hussein Onn Malaysia, 86400, Johor, Malaysia \\ E-mail: ${ }^{1}$ mhanif@uthm.edu.my; ${ }^{2}$ farhan@uthm.edu.my; ${ }^{3}$ shahreen@uthm.edu.my; ${ }^{4}$ norasri@uthm.edu.my \\ ${ }^{b}$ School of Industrial Engineering, Telkom University, Jalan Telekomunikasi No. 1, Bandung, 40257, Indonesia \\ E-mail: muharmanlubis@telkomuniversity.ac.id
}

${ }^{c}$ Department of Information System, Faculty of Industrial Engineering, Telkom University, Bandung, 40257, Indonesia E-mail:dedenw@telkomuniversity.ac.id

\begin{abstract}
Nowadays, the rapid enhancement of Internet connectivity and the recent progression of smartphone technologies lead to better smartphones quality towards video streaming activity. With the massive production of smartphone devices today, motivate studies of energy consumption behaviors to extend the smartphone device battery-life. Therefore, existing designs for smartphone devices occasionally lack energy-aware thus it need profiling optimization technique that reduces energy usage. Energy profiling in smartphone devices is one of the practical criteria for saving energy in smartphone devices during video streaming session. Energy efficiency features for smartphone devices, profiling and video content adaptation approach are the most critical parts for the energyefficient while streaming in course. However, the consideration of energy-aware profiling area has not yet been discovered widely. In this case, appointing promising approaches will be used to reduce energy consumption in the smartphone devices during video streaming session. A framework called PowerDoW will be benefited towards adding energy adaptation strategies. PowerDoW framework manage and utilize system profiling status to attain the entire streaming session activity and classify the streaming video format depending on the selective video parameter. Selection of the best quality depending on low energy usage will be determined in the profiling experimentation. The experimentations are based on the Android operating system in smartphone devicesinstrumentation setup testing by using PowerTutor application to measure energy consumption in real-time. The result indicates that PowerDoW framework can reduce a huge energy consumption by selecting suitable video content adaptation during video streaming session.
\end{abstract}

Keywords — video content-adaptation; energy-aware; energy profiling.

\section{INTRODUCTION}

The rapid development for both smartphone devices and application in today market shows that the user demand is growing steadily. Since the user demand is higher, most of the smartphone devices available on the market nowadays have glitch or development issues. In general, one of the issues is the lack of battery lifespan. Besides, the battery lifespan for existing smartphone devices is considerably limited for the video streaming propose.

The use of smartphone devices for streaming video content is pervasive. Hence, the energy consumption is very high to stream online video content [1]. It is due to many variations on online video attributes. Therefore, most of the consumer overlook that they are using high energy in a smartphone device without realizing it. Therefore, low energy in the video streaming session shorting the time streaming and leads to the unsatisfied user. Recently, many researchers have suggested new notions to prolong the battery capacity; yet, the development of battery technology is unpredictably and may not be available on the market shortly. It is crucial to extend the battery lifespan from a software viewpoint, and it becomes the main issues to the research community.

Even though there are a lot of enhancement already done in the previous studies that the problem of low battery 
lifespan is still intact and has become a key issue among smartphone users for video streaming online [2]-[6], [13]. Previously, some studies depicts that video content adaptation has a massive space for energy optimizationsthe optimization and alteration based on the modification of video content, application and algorithm [4], [7], [10]. Thus, the need of an energy-aware framework that satisfies user perspective and energy usage must be developed within a smartphone streaming environment. The analysis characteristics on smartphone preferences will be carried out before the development of framework can be considered. The framework must be enough to satisfy users needed and energy state, and consume less energy while streaming session is occurred. Nevertheless, in video content adaptation, it is difficult to produce a good video attribute that suits the energy and user satisfaction at the same time. This research study could provide the key finding for the issue of energy consumption, particularly the reliability and sustainability of smartphone device's battery power. Furthermore, this study would be beneficial to users and developers as this study generates the possible solution of the video attribute for the smartphone issues regarding on energy efficiency and content adaptation propose. Moreover, results from the experiments will be used to design a reliable energy model and content adaptation on a smartphone device. This study would provide necessary information on the different energy consumption using profiling and content adaptation approaches.

In the last era, there have been several energy-aware management of smartphone devices as well as handheld and electronic portable devices research topics introduced in the researcher's community. There is a huge quantity of related efforts that has been carried out concerning on video adaptation with energy efficiency [2], [8], [11]. Previous study [2] established the energy-hungry applications that might be knowingly inclined by the way how a device is functioned [7], [8]. Then, all the research related to energy efficiency based on content adaptation video streaming session. Relatively, content adaptation is the procedure of choosing, producing, or modifying content to outfit the user's preferences, consumption style, computing and communications environment, and usage context [11]. Although video communication is highly desirable, a primary curb in video content adaptation is the basic design architecture that batteries typically power the most mobile devices with limited capacity. Generally, content adaptation depicts the altering or modification content action to familiarize to the device or mobile capabilities [13]. Content adaptation is typically related to mobile devices that need distinct handling because of their inadequate processing power, in terms of screen sizing and constrained touch screen key functionality. The claimed that the video streaming at current incline to use more energy. In addition, previous study suggested on this subject there is a huge area to reducing energy consumption by altering application behaviour [4]. By using this approach, it is unnecessary to include apparatus on experiment while instrument approaches use certain measuring devices to capture power consumption in the smartphone devices. Software method using an internal battery interface (e.g. based on a battery sensor) and based on battery sensor, some researcher develops an application for energy profiler (e.g. Eprof, PowerScope, PowerTutor). Currently, energy-aware for video streaming content scheme has not been studied comprehensively and many researchers trying to create a suitable energy management policy within smartphone devices. Several studies [4], [7], examined to construct evaluation platforms to investigate the energy usage for video streaming. Another study [8] developed an application called EnVI to estimate energy consumption in video content adaptation. Some other studies mention that energy loss while video streaming, can be the main issues and affected energy efficiency [10], [14].

The primary motivation of studies was conducted based on user perspective towards energy usage on their mobile devices while the streaming session is occurring. Usually, energy usage on smartphone devices is always ignored by almost all user up until the battery is empty [12]. Even though users sometimes ignore the power consumption on their mobile devices, however, this is one of the options for energy efficiency for the user to reduce energy consumption on the streaming session. Video selection sometimes ignores by the user, and it leads to energy inefficiency. Also, measuring the energy consumption on smartphone devices will face the challenge from across different applications, and its energy estimation is uncorrelated with execution time, suggesting experimenting method profilers may help in optimizing applications for energy use [14]. Many researchers focus on many methods of frameworks design, and the energy consumption based on a particular function, including software and instruments, approaches that the smartphone device needs to become an energy-efficient tool for the user to continue using their equipment [2], [3], [6] [14]. Another perspective has been engaged in the development of energy efficiency. It is the battery performance, but, due to the technical, hardware and financial issue, it is still counterproductive to rely on the battery improvement to narrow the gap between the high energy consumption of multimedia processing and the limited battery capacity [13], [14]. Then, by reducing the quality of video streaming, power management. Thus, it may lead to the user's frustration. These previous researches were used to deliver energy-guideline propositions to video content for streaming. Unlike their last effort, our PowerDoW framework tries to select the suitable video content variation for energy efficiency within smartphone devices.

\section{MATERIALS AND METHOD}

To provision an energy variation for video content adaptation, this setup using the PowerDoW framework within a smartphone device with specific environmental features.

\section{A. The Framework of PowerDow}

As presented in Figure 1, the PowerDoW outline comprises two components: raw data collection and energy profiling policies.

Raw data collection is an element that collects video data preferences and energy usage.

The propose framework architecture aims to create a platform for intelligent monitoring of an energy usage that is 
customizable to user needs and offering the possibility to reduce energy consumption as low as possible. Next, all the energy consumption will be entered into the energy profiling state. Energy is catered by a simple app such as PowerTutor that collects data and using energy integration from devices itself. This is the reason of PowerDoW framework based on energy profiling by digitizing offset in the battery configuration in state of discharge. Using the power model implemented within an application estimates energy usage and the real energy on video content-adaptation. Energy profiling state getting into the energy-aware procedure state and performs the energy variation for energy efficiency. Then, video content selection will be selected with the best video variation for streaming purposes.

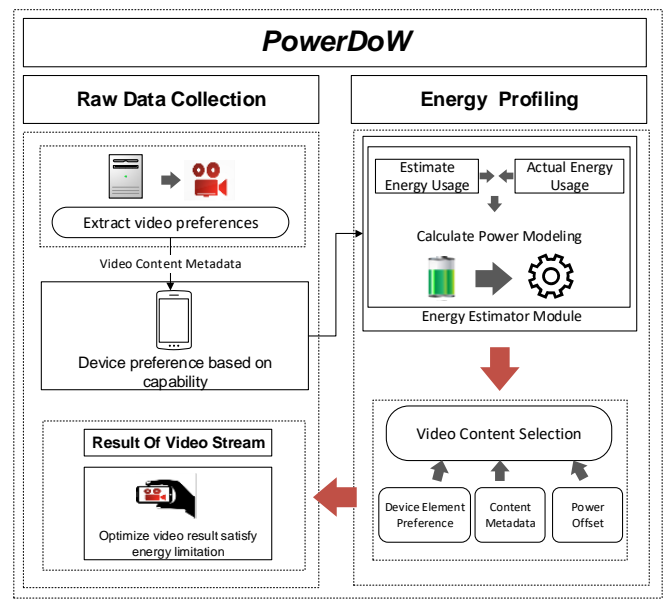

Fig. 1 the PowerDow framework

Since online streaming content contributes to draining the smartphone battery, the constraint needs to be characterized as the reason for energy consumption is infrequently deliberated at the early phase. Hence, the video contentadaptation where the video stream is available appropriately by select user expectation without having to worry about energy usage. Video content-adaptation is energy-efficient based on an impression of application adaption [8]; however, some of the attention on the bad user involvement triggered by inefficient battery life.

Since the user unaware about energy consumption, energy adaptation strategy is introduced. It is because we want to keep the smartphone in ready mode for telephone services and messages. Both factors are still the two foremost functions in smartphone devices. Lastly, in this framework strategy, it also extremely deliberates on user requirements; this experiment specifically states the best selection for user experiences within PowerDoW framework. Then, the Android platform with a certain system (Android 7, Nought) was chosen. Android OS has been chosen for this experiment platform because of reason e.i the availability of the variation of low devices specification and cost constraint.

Android's user across the world is more than other known operating systems (OS) available. The customization of the Android OS open source is easy and reliable in terms of device setup for experimentation. Another reason to choose the OS device is multiple brands in terms of cost, which is a lot cheaper, and this OS is various in its capability; screen size and camera, battery options. Since there are many variants available, a testbed for experiment result would be huge and easy to manipulate. One of the things that reliable about This OS device is the total device preferences and control over the device. That means if development for experimentation can be established by using Android Studio and simple application can be handle and improve relatively.

\section{B. Device Preferences}

Before an experiment is conducted to measure energy consumption, an appropriate device must be chosen. There are many constraints in choosing suitable smartphone devices for the experiment. Nonetheless, there is some reliable consideration in terms of usage and compatibility. First, we determine all smartphone device specification, and it can be defined as $(\delta s)=\left\{x \delta_{v 1}, x \delta_{v 2}, x \delta_{v 3} \ldots . . x \delta_{v n}\right)$. Where $x \delta_{v n}$ is the variation of low, middle and high specification smartphone devices; so the device specification order is $(\delta s)$ $=\left\{L \delta_{1}, L \delta_{2}, L \delta_{3}, M \delta_{1}, M \delta_{2}, M \delta_{3}, H \delta_{1}, \quad H \delta_{2}\right.$ and $H \delta_{3}$ ) respectively. $\mathrm{L} \delta_{1}$ represents for low device capability, $\mathrm{M} \delta_{1}$ for medium and $\mathrm{H} \delta_{1}$ for high specification in smartphone selection. Moreover, all devices that have been chosen are capable of being arranged in terms of the software part.

\section{Energy Estimator Module}

To support the energy efficiency in the smartphone devices, we implement an energy estimator module within the energy profiling state. The energy estimator module contains three parts: estimate energy usage (state collector), determine actual energy consumption and calculate power modelling. First, actual energy from power offset $\left(P_{\text {offset }}\right)$ determines the energy baseline and estimate by using a power profiling model $\left(P P_{\text {Model }}\right)$. $P_{\text {offset }}$ is set as $P_{\text {offset }}=\chi c p u$ $+\chi$ display $+\chi$ gps $+\chi$ audio $+\chi$ network $\ldots \chi(n)$. Initial $\chi(n)$ total elements are comprised within power offset and combine with $\sum_{j=\delta_{s(l)}}^{\delta s(n)}(\chi \delta n)$ To calculate the actual power modelling for video adaptation used in the experiment later. Equation 1 shows the power profiling features in experimentation devices.

$$
P P_{\text {Model }}=p_{\text {offset }}+\sum_{j=\delta_{s(l)}}^{\delta s(n)}(\chi \delta n)
$$

Where, $P_{\text {offset }}$ elements including all the smartphone device component corresponding to energy baseline $(e \beta)$ such as battery capacity, a power gauge that cannot be turned off because it is responsible for OS kernal [14]. It is also call system idle and ready mode power consumption. $\delta$ s can be defined as devices energy metadata (WiFi, brightness). $j=\delta s_{(1)}$ is initial $\delta s$ that is a start for estimate energy consumption. $\chi \delta n$ total device involves elements within the smartphone device. Particularly, the compose power profiling model will be used to prompt energy adaptation directly later.

\section{Video Content Selection}

Video content selection component estimate the capability of video content streaming. The experiment selects the most recognize video formatting available nowadays, since the video format such as .mp4, .mkv, .avi are easier to manipulate, but it difficult to comprehend currently [5]. Most of the video is available to use progressive for videos streaming in smartphone devices. 
This video quality format type is a standard video format for smartphone devices that exist nowadays, and it referring to the ITU standards [13]. The support video encoding parameters, H.264 Baseline Profile codec is used. Since the progressive scanning method is used, the video format for low-quality video can be defined as $144 \mathrm{p}, 240 \mathrm{p}$, whereas for medium quality is $360 \mathrm{p}$. Standard quality video can be defined as $480 \mathrm{p}$, and high-quality video format is $720 \mathrm{p}$. The highest video quality is full high definition video with 1080p. Normally, a video content adaptation (VCA) is the action of converting or transforming video content to fit a device capability. We alter these video formats into numerical order for experimental propose. $\mathrm{VCA}=v c_{(1)}, v c_{(2)}, v c_{(3)}, v c_{(4)} \ldots .$. $v c_{(n)}$, where $v c_{(1)}$ is $144 \mathrm{p}, v c_{(2)} 240 \mathrm{p}, v c_{(3)} 360 \mathrm{p}, v c_{(4)} 480 \mathrm{p}, v c_{(5)}$ $720 \mathrm{p}$ and $v c_{(6)} 1080 \mathrm{p}$. This VCA can be added to equation 1 and compare with baseline and device energy.

To simulate the video content adaptation, VCA attributes will be inserted within the equation 2 .

$$
P P_{\text {Model }}=\sum_{i=v_{c(1)}}^{v c(n)}(\chi v c)-\left[p_{\text {offset }}+\sum_{j=\delta_{s(1)}}^{\delta s(n)}(\chi \delta n)\right]
$$

Equation 2 shows the additional video content preference equation to the previous equation $1 . v c(n)$ illustrate the maximum number at video metadata preferences that can be implemented inside the smartphone devices while $i$ is defined as the variable of video metadata preferences (brightness, resolution, frame rate). Video metadata preference can be classified as raw video streaming content that can be extracted as content metadata. We can deduct the energy from actual usage from $e \beta+p_{\text {offset }}$ and total usage of energy consumption to find the actual energy consumption result for VCA.

The implementation of the PowerDoW framework for experimentation is not only on Android 7 but with some updated version of smartphone device. Before running an experiment on video streaming for energy consumption, $\delta s$ instrument will be divided into three groups of the tier where all the characteristics of smartphone devices are classified; low, medium, and high specification devices. The setup begins with the measurement of the power offset during an idle session on $\delta s$. Within the idle state, all the features of the application and operating system are disabled. Only standby mode is enabled for video streaming. Since the measurement is done by using PowerTutor software, all the energy states such as CPU load, brightness and even single application energy consumption appear separately and can be measured easily. PowerTutor uses in smartphone Android application .apk because of it is a small application tool for Android device and its functionality to profiling data from smartphone devices power status in the real-time state [14]. Energy profiling is implemented which certainly have three functions: gathering/collecting data info, assessing video energy usage and handling energy usage of the smartphone devices. The experiment measurement relies on miliampere/hour $(m A h)$ depending on battery capacity.

The experimentation will determine energy usage on these classified smartphone devices. All three specification devices are run through with 20 cycles of experimentation to calculate the average of the results. Firstly, a video sample was uploaded into the server with the .mp4 extension format.
Then, by using a Wowza server as the establish server for video streaming, the video sample is in standby mode. Furthermore, the brightness in device capability is set to high $(50 \%)$ of the brightness setting. To avoid disruption on connection, a stable WiFi connection is established throughout the experiment. Figure 2 depicts the result of baseline level based on the power offset of smartphone devices.

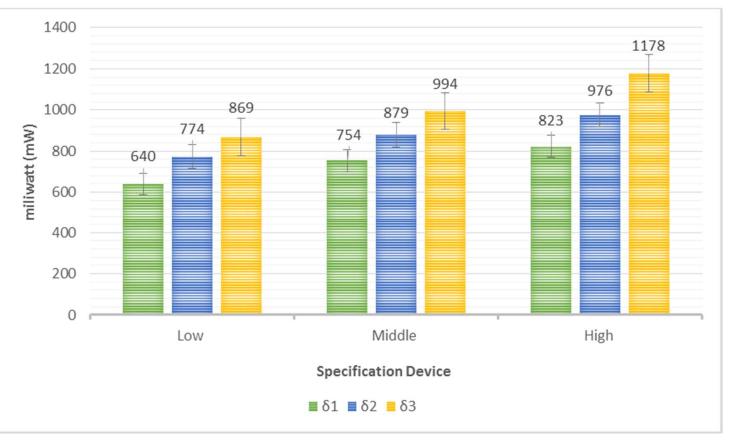

Fig. 2 Power Offset Baseline of Smartphone Devices

Based on $p_{\text {offset }}=\chi c p u+\chi$ display $+\chi$ gps $+\chi$ audio + $\chi$ network.... $\chi(n)$. Result of the experiment; based on the energy $\quad e \beta(L \delta)=\sum\left(\frac{\beta b a s e\left(T_{0} t_{-} L \delta\right)+\alpha\left(T_{0} t_{-} L \delta\right)+W\left(T o t_{-} L \delta\right)}{n \beta+n \alpha+n W}\right)$ Where $e \beta(L \delta)$ is the average energy consumption of low $\delta s$ energy baseline.

pbase is energy from $e \beta . \alpha$ is defined as The resulting yield $38.9 \%$ energy usage for $p_{\text {offset }} . e \beta(M \delta)=40.8 \%$ and $e \beta(H \delta)=39.8 \%$. So to obtain the energy baseline for minimum efficiency in devices is $e \beta(L \delta)=\frac{e \beta(L \delta)+e \beta(M \delta)+e \beta(H \delta)}{e \beta(n)}$ and a total of result shows the percentage of energy consumption for baseline threshold is $\approx 40 \%$ of smartphone energy capacity. All these baseline experiments setup combine with hardware capability components, and the whole process can be demonstrated for the different variety of discharge level while streaming HD video [12], [13]. The experiment result shows the slightly linear drop on the graph, however within area $60 \%$ to $40 \%$ of battery usage, a sturdy curve is revealed. After baseline energy experiment was conducted, the next experiment is profiling the energy consumption based on the $\delta s$ setup.

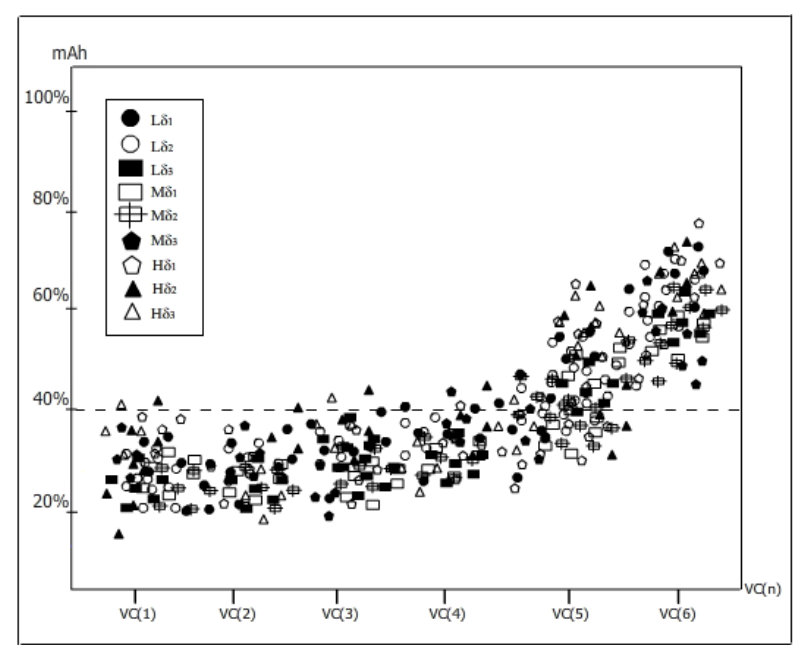

Fig. 3 Energy profiling for video content adaptation in selected smartphone devices 
Figure 3 depicts a scatter plot graph for both video content adaptation experiments and energy baseline nominal line usage by using energy profiling approaches in smartphone devices. This figure also shows the energy $(m A h)$ versus video content adaptation $v c_{(n)}$. All smartphone device specification $\delta s$ are undergone with this experiments including video content adaptation $\left(v c_{(1)}, v c_{(2)}, v c_{(3)}, v c_{(4)}\right.$, $\left.v c_{(5)}, v c_{(6)}\right)$. The result shows the dissemination of scattered energy data. Fundamentally, this experiment is to show the result of discharge time or drainage of power consumption without any other interruption. After the baseline experiment is completed to simulate the energy profiling for video content, all the devices must go through the profiling experiment to obtain energy consumption for video streaming session. Energy data are scattered all over the graph. However, the data depict an ascending trend from lower energy consumption to higher energy consumption within smartphone specification devices.

From this data distribution, it leads to over-fitting of data [9]. Besides, over-fitting can happen depending on two factors; the complexity of the data and the generating amount of training dataset [10]. From energy baseline experimentation, the maximum energy consumption of at least $40 \%$ of the battery of the devices. It can be converted to the rule-based method in order to determine or classify the most efficient $V C A$ in term of energy consumption. Almost half of the result is above the region of $40 \%$ energy usage, and the other half are below $40 \%$. This result determined the efficiency of the minimum requirement of energy consumption.
After defining the variation of energy usage and VCA, the next step is to determine the number of energy-efficient variation. An energy-efficient variation is a combination of both experimentations between energy baseline experiment and energy efficiency based on the energy profiling algorithm. Then, the next step is to determine the VCA attribute that can be a substitute to instances and its figure setting. From the experiments, the VCA can be classified as follows:

$$
V C A=\left\{v c_{(1)}, v c_{(2)}, v c_{(3)}, v c_{(4)}, v c_{(5)}, v c_{(6)}\right\}
$$

Classification of $V C A$ instances by sorting them down to the list and then the leaf node trees represent disjunction ${ }^{\wedge}$ of conjunctions of constraints on the attribute values of instances. Replacing if rule meets the condition of the energy-efficient, then the value will be resulting in weather is pruning or no pruning as follows:

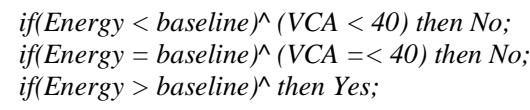

Then the value is map into the tree with a certain condition in order to compare the value for possible energyefficient variation. Figure 4 shows the mapping of video content adaptation tree for possible energy-efficient variation. $V C A$ is compared with the condition in rule post-pruning. $i$ represent the starting phase of the post pruning tree while $\mathrm{c}$ is a list of VCA. $c$ illustrates an energy consumption of smartphone specification devices while $b$ is the baseline energy experiment that already done earlier. There are six variation of video content adaptation $\left(v c_{(1)}, v c_{(2)}, v c_{(3)}, v c_{(4)}\right.$, $\left.v c_{(5)}, v c_{(6)}\right)$.

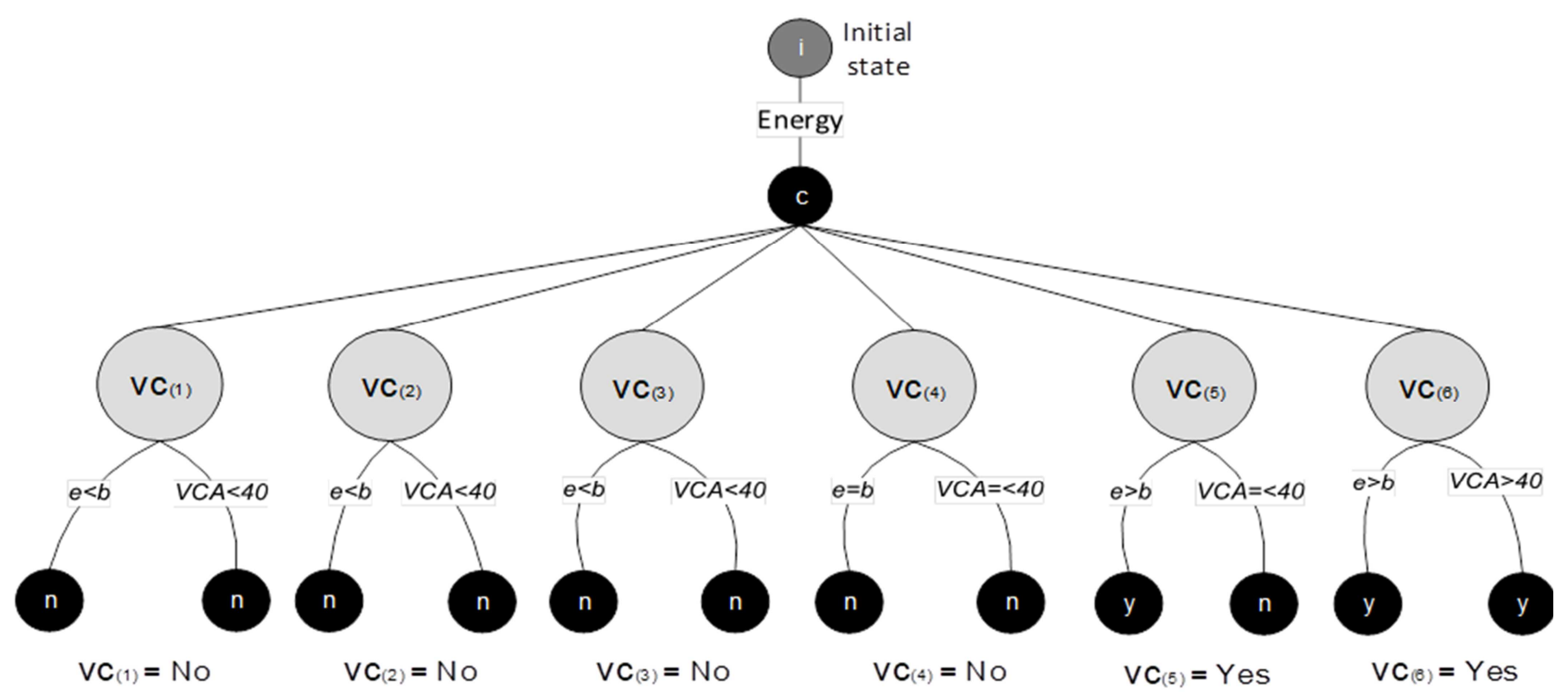

Fig. 4 video content adaptation (VCA) tree for possible energy efficient variation

This tree of possible variation has two conditions to meet in order to pass the pruning level. First is the baseline rule, and the second is the $V C A$ rule. The baseline rule, all $V C A$ energy on devices capability requires not more than 40 per cent of energy baseline in order to prune the $V C A$ node.
Furthermore, VCA rule depends on energy profiling experimentation being equal or not more than 40 per cent of energy usage. If both requirements are met on $V C A$, the rule post-pruning method will be implemented. In addition, from Figure 3, display the pruning will be on the $v c_{(5)}$ and $v c_{(6)}$ of 
$V C A$ because it meets the requirements of rule post-pruning method. That leaves the rest of $V C A\left(v c_{(1)}, v c_{(2)}, v c_{(3)}, v c_{(4)}\right)$ using the less energy consumption while streaming video content with a possible energy-efficient variation. In the user viewpoint, the possible video variation could be improved in term of software or application used.

However, the result simulated within control environments and scenarios and using a wireless connection. In a real situation, it may cause more energy usage, while streaming services are running within smartphone devices.

\section{A. PowerDoW Case Study}

To confirm these approaches, we done some of the clusters of experiments alongside with $V C A$ without power profiling that adapts the PowerDoW framework that we modify. We run on certain smartphone devices for experimentation proposes following the control environment experimentation setup. First, the experiment is run on smartphone devices to test devices reliability in terms of energy consumption. Experimentation will be repeated twenty times for every device. Understanding power consumption measurement of energy profiling is the first step to improve its power efficiency. Ensuring a consistent operating environment is essential for obtaining useful power data of the application.

In the preliminary testing experiment, the testing device that setup for jumper cable on smartphone battery of Samsung Galaxy smartphone device. In this testing, the control environment is established. In figure 5, shows the smartphone device profiling experiment testing and setup. One smartphone device, Samsung Galaxy S2 running on the Android operating system for the experiment setup connect to the multimeter for energy measuring while experimentation is executed. Then, the setup connection type is set to only $\mathrm{WiFi}$ (wireless connection), while other sensors that may interrupt energy usage are turned into offline mode.

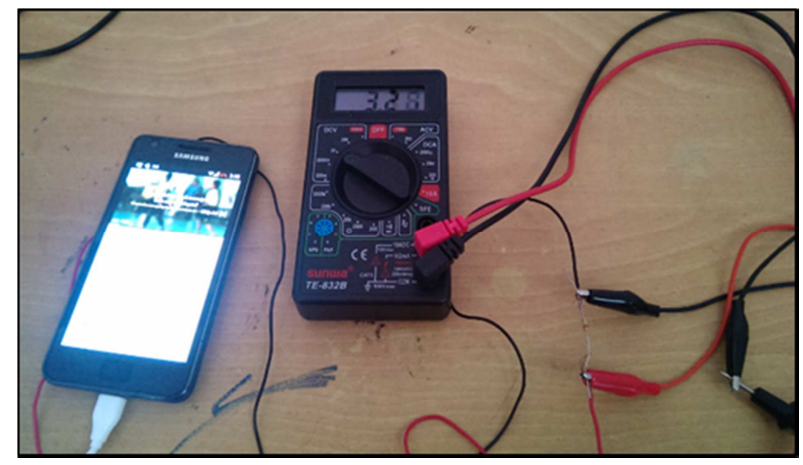

Fig. 5 The energy result of VCA using profiling adaptation

A test experiment uses a video player application in a smartphone device (e.g. YouTube and Vimeo). The video characteristics as follows the format is 480p, and the duration is 10 minutes per one reading. Device preference is set to maximize the brightness of the display and audio setting. Testing requires the video stream to play at every 10 minutes, and reading is taken by multimeter until the battery completely drained. These experiments are repeated 10 times in order to get an accurate average result of energy usage of smartphone devices. Figure 6 shows the experiment results.

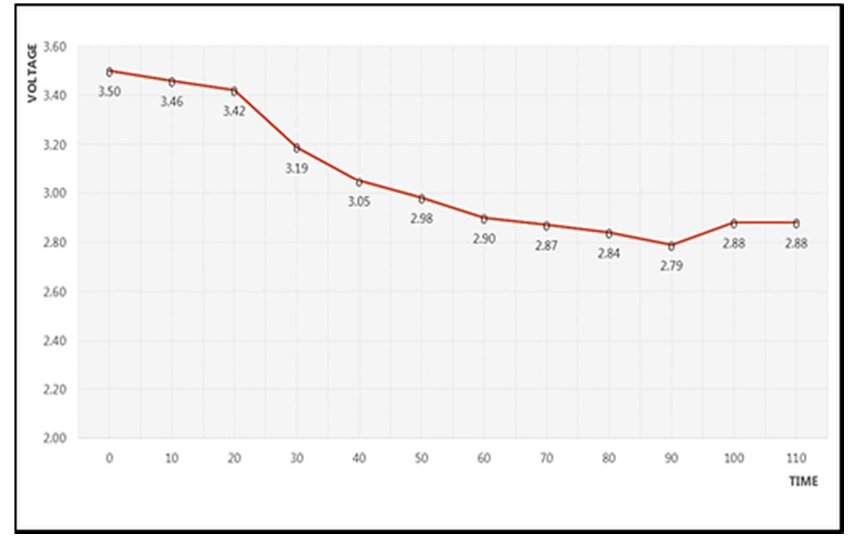

Fig. 6 Experiment result of preliminary testing on the device

After that result calculated in order to obtained mean error for device selection. This preliminary testing main propose is to illustrate the reliability of smartphone devices battery capability. Some of the devices use higher energy consumption due to battery capability, device preferences such as cpu power and network capability and another obvious inefficiency within smartphone devices such as overheat. Nevertheless, within this study, it did not cover another inefficiency that occurs in smartphone devices. The higher the error result on devices means, the less consistent the result for experimentation later.

With a similar setup, our result indicates that there is an average of $20 \%-30 \%$ energy consumption possibilities can be saved and energy misused by video streaming application. We use a modified Android video player: altered media player based on the PowerDoW framework of the Android platform OS. Initially, for this case study, we stream a video with similar video content with power profiling model and without a power profiling model-both testing running on the video application apk. The testing begins with energy measurement using PowerTutor and energy (mAh).

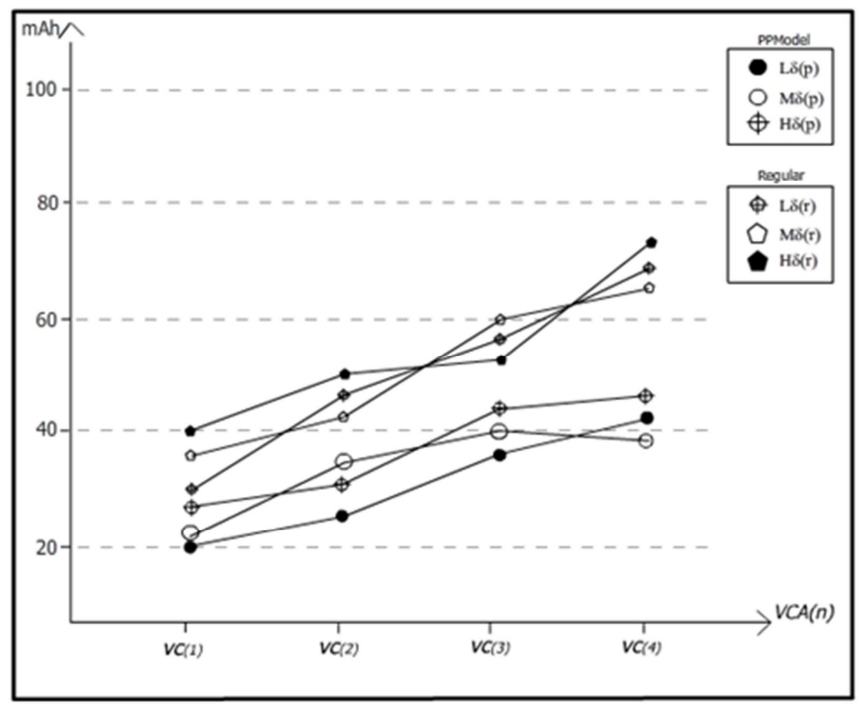

Fig. 7 Energy result of VCA using profiling adaptation

Figure 5 illustrates an energy consumption result when VCA profiling in active adaptation mode. By using a similar setup from earlier experiments from e $\beta$ and $p_{\text {offset }}$. This will be weakening the intention required for processing of video data. From Figure 5 shows this technique is operational to 
reduce the energy usage on mobile devices. Averagely, about $26.8 \%$ of energy usage might be preserved. The equation $e \beta c a s e=\sum\left(\frac{(e L \delta)+(e M \delta)+(e H \delta)}{e \delta(n)}\right)$ Where e $\beta$ case is an experiment of energy-based of the case study, eL $\delta, \mathrm{eM} \delta$ and $\mathrm{eH} \delta$ is total case study $\delta$ s energy consumption. In Figure 7 , the result shows the average energy consumption in $\delta$ s. That average of power profiling model used less energy rather than without a power profiling model. With power profiling model, the energy usage significantly reduces almost $28 \%$ differ from regular without power profiling usage. It uses almost $30 \%$ of energy consumption on smartphone devices. The energy usage of the entire system loses when the video apk initiates energy adaptation approaches. This framework can be implemented within the mainstreaming application, such as Youtube and Vimeo. For this framework to work with the application there should be some of the alterations on how video streaming can be manipulated. Moreover, it will sit on the server-side of mainstreaming server if available. This approach is reliable as the experiment process used a similar concept. In addition, by setting an experiment on energy efficiency show the significance reduces energy consumption. Since PowerDoW framework is a set of process and instruction, hence, it also possible to implemented and embedded on OS as well.

\section{CONCLUSION}

Generally, in any research being conducted, it will have some constraints. In this paper, the main limitation is to form a suitable energy model that will fit with any devices, scenarios, and environments. This research tried to proposed video content adaptation and implemented into the framework of PowerDoW approaches. Basically, there is a lot of improvement that can be made in order to enable the adaptation of the energy function. Next, this section comprises several improvements that can be implemented within this area. User Quality of Experience (QoE). Essentially, user behaviours and reaction play the main role when it comes to reducing energy consumption in smartphone devices. The user QoE that can expect user actions and determine the best solution towards energy management and user preferences. User QoE ought to estimate the user desire in order to give the best outcome for their video streaming that satisfy both QoE and energy in the smartphone device. In future work, research on user QoE is highly necessary or compulsory and must be considered for fully designing the best energy-aware policies for video streaming application or session. Policies of Energy-aware. Since more update on Android OS, it is difficult to design energy-aware policies in order to support energy adaptation. We need to create a universal profiling algorithm that caters to both energies on video content adaptation within an application device. This objective requires the policies to not only be good at lessening energy consumption for video content streaming, nevertheless, also considering the variance of each video application. Remote content adaptation engine provides a read-time choice measurement for media prediction in video streaming. In order to find the best solution for video attribute, it must be triggered automatically to find good video attributes being implemented in the server.

\section{REFERENCES}

[1] Amine, D., Elmiligi, H., Gebali, F., Energy Bugs in Mobile Devices: A Survey. IEEE Pacific Rim Conference on Communications, Computers and Signal Processing (PACRIM) 2015.

[2] Ballesteros, L.G.M., Ickin, S., Fiedler. M., Markendahl, J. Energy Saving Approaches for Video Streaming on Smartphone based on QoE Modeling. 13th IEEE Annual Consumer Communications \& Networking Conference (CCNC), 2016.

[3] Dragiü, L., Hofman, D., Kovap, M., Žagar, M., Knezoviü., J. Power Consumption and Bandwidth Savings with Video Transcoding to Mobile Device-specific Spatial Resolution. 9th International Symposium on Communication Systems, Networks \& Digital Sign (CSNDSP) 2014.

[4] Ismail, N.M., Ibrahim, R., Fudzee, M., F. A Survey on Content Adaptation Systems towards Energy Consumption Awareness. Hindawi Publishing Corporation, Advance in Multimedia Volume 2013 - 8page, 2013.

[5] Khan, S., Schroeder, D., Essaili, E., A., Eckehard, S. Energy-efficient and QoE-driven adaptive HTTP streaming over LTE. IEEE WCNC'14 Track 3 (Mobile and Wireless Networks) 2014.

[6] Zheng, Q., Du, H., Li, J., Zhang, W., Li, Q. Open-LTE: An Open LTE Simulator For Mobile Video Streaming, IEEE International Conference on Multimedia and Expo Workshops (ICMEW) 2014.

[7] Aqil. A., Ahmed O. F.., A *, Srikanth., V. K., *, Papageorgiou. Streaming Lower Quality Video over LTE: How Much Energy Can You Save? IEEE 23rd International Conference on Network Protocols 2015.

[8] Oche, N., Saleem, N. B. Go Green with EnVI: The Energy-Video Index. IEEE International Symposium on Multimedia 2015.

[9] Fudzee, M., F, and Abawajy., J. A protocol for discovering content adaptation services. Algoriths and Architectures for Parallel Processing (2011). pp. 235-244.

[10] Dung, T., N, Ho, T., B., Shimodaira., H. A Scalable Algorithm for Rule Post-pruning of Large Decision Trees. Advances in Knowledge Discovery and Data Mining (2001) pp 467-476.

[11] Fudzee, M., F, Abawajy., J. Management of Service Level Agreement for Service-Oriented Content Adaptation Platform. Network and raffic engineering in emerging distributed computing applications (2012) pp 21-42. ISBN- 1466618892.

[12] ITU-T Standards for Video Encoding - "Subjective video quality assessment methods for multimedia applications," ITU-T Recommendation P.910, April (2008).

[13] Ismail, M., N, Fudzee, M., F, Ibrahim., R., Jofri., M., H. Video Streaming Energy Consumption Analysis for Content Adaption Decision-Taking. Journal of Telecommunication, Electronic and Computer Engineering (2017). ISSN: 2180-1843.

[14] Tarkoma. S., Sikkinen. M., Lagerspetz. E., Xiao. Y., Smartphone Energy Consumption: Modeling and Optimization. University Printing House Cambridge 2014. 